\title{
Gelatin based microfluidic devices for cell culture $\dagger$
}

\author{
A. Paguirigan and D. J. Beebe* \\ Received 9th December 2005, Accepted 12th December 2005 \\ First published as an Advance Article on the web 18th January 2006 \\ DOI: 10.1039/b517524k
}

\begin{abstract}
We have developed a technique for fabricating microfluidic devices from gelatin using a natural crosslinking process. Gelatin, crosslinked with the naturally occuring enzyme transglutaminase is molded to produce microchannels suitable for adherent cell culture and analysis. The autofluorescence of the material was shown to be minimal and within the range of typical background, ensuring utility with analyses using fluorescent dyes and labels would not be affected. Also, normal murine mammary epithelial cells were successfully cultured in the microchannels. The morphology of these adherent epithelial cells was shown to be significantly different for cells grown on rigid tissue culture plastic in either macro- or microscale cultures (even in the presence of a surface coating of gelatin) than those grown on the flexible crosslinked gelatin microchannels. Using these devices, the effects of both the extracellular matrix and soluble factors on cellular behavior and differentiation can be studied in microenvironments that more closely mimic the in vivo environment.
\end{abstract}

\section{Introduction}

While microfluidics has shown considerable promise as a tool for studying cell biology, the potential for microfluidics to create more in vivo-like in vitro environments (or, in visio environments) is still largely untapped. It is becoming clear that the scale of the microenvironment provided by microchannels is an important biological parameter. Microchannels have been used for several steps in the in vitro production of embryos typically either matching or improving the performance of previous methods. ${ }^{1,2}$ Insect cell cultures as well have shown very different dimension dependent growth kinetics in microscale cultures as compared to macroscale flask cultures. ${ }^{3}$

However, much of the previous culture work in microfluidics has focused on non-adherent (e.g., embryos, insect cells) or 2-D adherent cell culture in the absence of extracellular matrix. While the effects of the extracellular matrix are of critical importance to in vivo tissue function, ${ }^{4,5}$ the integration of extracellular matrix (ECM) materials into microfluidic systems is just emerging. ${ }^{6,7}$

We have fabricated microdevices which can allow for control over the soluble microenvironment, while also providing a more in vivo-like substrate (flexible protein gels) as a step towards an in visio environment for cellular analysis. We present a fabrication protocol to produce enzymatically crosslinked gelatin microdevices composed of natural components, that are sterile and suitable for cell culture. Gelatin is a derivative of collagen, one of the most common extracellular matrix proteins. These devices were tested for their fluorescence properties to ensure that any autofluorescence of the material itself will not limit its utility while using fluorescent dyes for cellular analysis. Also, we have explored the potential

2142 Engineering Centers Bldg., 1550 Engineering Dr., Madison,

WI 53704.Ph.,USA. E-mail: djbeebe@wisc.edu; Tel: (608) 262-2260

$\uparrow$ Electronic supplementary information (ESI) available: Fig. 5 and 6 and experimental details. See DOI: 10.1039/b517524k for mammary epithelial cell culture in these devices and compared the cell morphology at multiple time points between macro- and microscale cultures on traditional rigid surfaces and on flexible gelatin gel substrates.

\section{Background}

The in vivo extracellular matrix is an important regulator of a cell's microenvironment and thus cellular behavior and expression. The extracellular matrix is known to be a continuous source of both mechanical support and chemical information, either soluble or via sites of interaction with cellular surface receptors and complexes. Observations of differentiation, morphology and gene expression due to changes in microenvironment have provided evidence that the microenvironment plays a major role in determining cell fate and behavior. ${ }^{8}$

Traditional macroscale and current microscale in vitro adherent cell culture involves using undefined media over a thin layer of cells grown on rigid surfaces (e.g. treated polystyrene). In these culture conditions, ECM components are typically either absent or present only as a thin layer of protein adsorbed onto a rigid surface. Neither a 3-D extracellular matrix nor physiological levels of soluble factors are present in this type of culture, resulting in a significantly altered microenvironment from that experienced in vivo.

A basic limitation of most in vitro assays of cell behavior or expression is the inability to correlate results to in vivo behavior because of the differences between the in vitro and in vivo microenvironments. This limits the ability to accurately understand regulation mechanisms and differentiation pathways occurring in vivo using in vitro assays, thus limiting the development of potential therapeutics for cancer or degenerative diseases. More in visio microenvironments for cellular analysis will improve the accessibility of in vivo responses of cells and tissues to stimuli that are impossible to study effectively using current in vitro methods. 
One major difference between the macroscale and microfluidic cultures is the importance of diffusion at the microscale. $^{9-11}$ In the absence of convective flow, local concentrations of components added to the media, secreted by the cells or present in surface treatment materials, along with those components remaining from fabrication, can have more pronounced effects on cellular response than in traditional cultures. The turbulent mixing and much larger volumes found in traditional macroscale cultures allow these components to be diluted throughout the entire volume and thus minimize their effects on the cells. Currently, biocompatible polymers such as poly(dimethyl siloxane) (PDMS) have been widely used for microscale cell assay and culture due to its gas permeability and inertness. Cultures in microdevices fabricated using PDMS are generally 2-D cultures on traditional tissue culture plastic. In these cultures, PDMS has no bioactivity, and simply serves as a mechanism to hold in culture medium, not a substrate for culture.

Recent work creating natural polymers has employed a purified natural enzyme, microbial transglutaminase (mTG), to crosslink gelatin and other cell adhesive molecules to create more biocompatible, injectable tissue scaffolding ${ }^{12}$ or adhesives. ${ }^{13}$ Preliminary results involving micromolding of gelatin using transglutaminase have also been produced. ${ }^{14}$ Microbial transglutaminase is currently used in commercial food applications to crosslink proteins found in milk and meat to improve texture or for molding. Also, it has been used to produce biodegradable or edible films, or bioplastics ${ }^{19}$ and has been shown to improve material properties of solid protein materials such as soy proteins for these purposes. ${ }^{20}$

In vivo, transglutaminase is present in several forms in tissue, blood, ECM and on cell surfaces. All proteins in the transglutaminase family have transamidating activity, and catalyze the formation of $\mathrm{N}-\varepsilon$ - $(\gamma$-glutamyl)lysine protein crosslinks. Members of the transglutaminase family are expressed in many tissues, and have many functions in processes such as posttranslational modification of proteins, cell adhesion and migration, extracellular matrix remodeling and blood clotting at wounds (fibrin polymerization). ${ }^{1,17,18}$ The transamidating activity of this enzyme is relatively evolutionarily conserved, and microbial transglutaminase (mTG) has a similar transamidating ability as the mammalian versions.

Enzymatically crosslinked gelatin gels have been shown to be biocompatible to both human and bacterial cells and both have been crosslinked into three dimensional matrices of gelatin with good cell survival. ${ }^{12,15,16}$ Gelatin/mTG biopolymer systems have a distinct advantage over previous synthetic hydrogel systems because of their superior biocompatibility and low costs. For these reasons, we have chosen gelatin and microbial transglutaminase as a material for our microdevices. Fabricating microdevices from collagen itself would be cost prohibitive. The high cost of purified collagen and the amount of material needed makes the ability to use gelatin in place of collagen for cell culture very important.

\section{Materials and methods}

The fabrication method used to create microfluidic channels in crosslinked gelatin is summarized in Fig. 1. Negative (a)

Photoresist

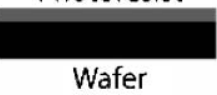

(d)

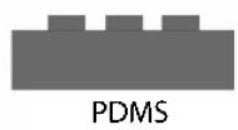

(b)

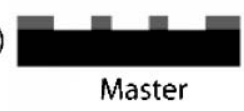

(e)
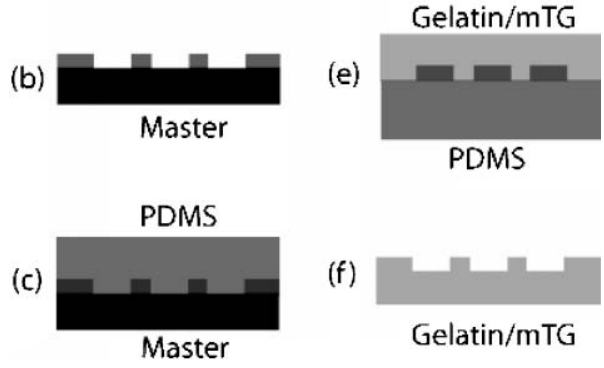

(f)

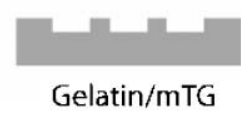

Fig. 1 Schematic of the molding procedure. SU-8 100 photoresist is spin coated onto silicon wafers (a), and UV exposure through a photomask and developing produces a master (b). PDMS is then cured on this master (c), and used as a mold for gelatin (d). The gelatin and enzyme solution is then poured over the reusable, flexible PDMS mold (e), and the polymerized device is then pulled off the mold (f).

photoresist, SU-8-100 (Microchem) was spin coated onto silicon wafers using the standard Microchem protocol for $250 \mu \mathrm{m}$ feature depth. Photomasks were used to produce the negative of the PDMS mold desired. PDMS (with 1:10 curing agent to prepolymer weight ratio) was then cured on the master (at $85{ }^{\circ} \mathrm{C}$ for 90 minutes), removed and used as a reusable mold for the gelatin/mTG solution with no further treatment.

The gelatin/mTG prepolymer mixture was made using an $11 \mathrm{wt} \%$ gelatin (Type A from porcine skin, 300 bloom, Sigma) solution in $1 \times$ Dulbecco's Phosphate Buffered Saline (DPBS). This solution was heated (not higher than $70{ }^{\circ} \mathrm{C}$ ) to solubilize the gelatin and $0.02 \%$ chloroform was added to sterilize the solution. The microbial transglutaminase (Activa-TI, Ajinomoto, Inc., activity of approximately $100 \mathrm{U} \mathrm{g} \mathrm{g}^{-1}$ of mTG according to the manufacturer) was dissolved in $1 \times$ DPBS using the appropriate mass of $\mathrm{mTG}$ to give $10 \mathrm{U} \mathrm{mTG}$ $\mathrm{g}^{-1}$ of gelatin. The final concentrations of the prepolymer mixture used in molding were $10 \mathrm{wt} \%$ gelatin, with $10 \mathrm{U}$ mTG $\mathrm{g}^{-1}$ of gelatin. The resulting mixture was poured over the PDMS molds and incubated at $37{ }^{\circ} \mathrm{C}$ for 5 hours. Initial gelation occurred within 5 minutes, but the mixture was incubated longer to obtain higher degrees of crosslinking and higher stiffness to facilitate removal from the molds. After incubation the material was cooled to $4{ }^{\circ} \mathrm{C}$ and removed from the PDMS mold. These gels were then heat treated in $1 \times$ DPBS for 30 minutes at $65{ }^{\circ} \mathrm{C}$ to heat inactivate and remove the enzyme and dialyze any remaining chloroform from the gels.

Before use, crosslinked gelatin gel microchannels were placed with the molded side up and a thin $(500 \mu \mathrm{m})$ membrane of PDMS with ports cored at the appropriate locations laid over the top to form a complete fluidic channel (see Fig. 2). The PDMS membranes adhere reversibly to the surface of the gelatin and seal the channels.

To test whether these crosslinked gelatin gels are suitable for microscale cell culture, a variety of conditions were tested. As this research is directed towards studying primary mammary 


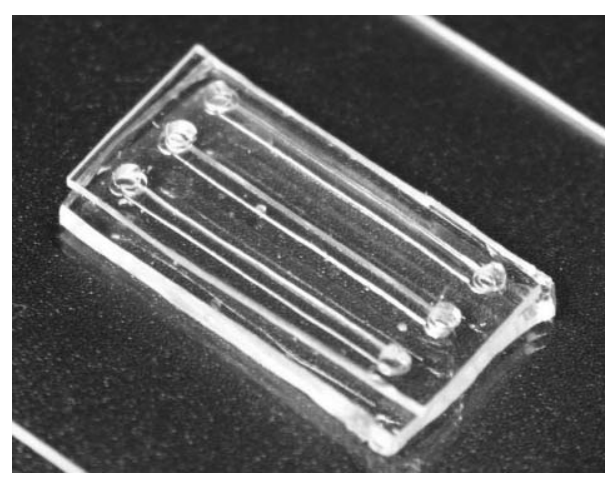

Fig. 2 Transglutaminase crosslinked gelatin device with a thin PDMS membrane as a top to create access ports. Channels molded in this device are $1,000 \mu \mathrm{m}$ wide, $20 \mathrm{~mm}$ long and $250 \mu \mathrm{m}$ deep.

epithelial cells for breast cancer research purposes, we chose to use an established normal murine mammary epithelial cell line (NMuMG) for analysis of the material. For treatment before culture of NMuMG cells, molded gelatin gels were soaked in Dulbecco's Modified Eagle's Medium (DMEM) overnight to prevent leaching of media components into the gels during the culture.

Traditional flask culture was performed using DMEM, supplemented with $10 \%$ fetal bovine serum (FBS) and $1 \mu \mathrm{g}$ $\mathrm{mL}^{-1}$ insulin. Cells were trypsinized to remove them from the flask surface, diluted in fresh media and then seeded into the different experimental conditions.

As a control, cells were seeded into PDMS channels of the same dimensions as those of the gelatin channels. These were molded in a similar way as the gelatin molds using a master with the same feature thickness but inverted. The PDMS devices were UV sterilized and humidified prior to placement on tissue culture treated polystyrene surfaces for cell culture. Gelatin coating of the polystyrene substrate in these PDMS microchannels was done using $0.01 \mathrm{wt} \%$ gelatin in deionized water, which was allowed to remain in the channels overnight at $37{ }^{\circ} \mathrm{C}$, a standard surface coating protocol. The gelatin solution was then washed out prior to cell seeding with fresh media.

Optical spectroscopic measurements were made using a conventional laboratory spectrofluorometer (Fluorolog-3, JY Horiba, Inc., NJ) with a fiber-optic probe coupled to the sample compartment (F3000, JY Horiba, Inc.). A detailed description of the instrument is provided elsewhere. ${ }^{21}$ Fluorescence spectra were measured at four excitation wavelengths: $320,490,550$, and $650 \mathrm{~nm}$ and the excitation and emission wavelength band passes were 5 and $10 \mathrm{~nm}$, respectively. Each fluorescence spectrum was sampled in $5 \mathrm{~nm}$ increments, and the integration time was 0.5 seconds per wavelength. The high voltage was set to $950 \mathrm{~V}$ for all measurements. Probe depth of the fiber optic probe was much larger than the thickness of the samples (approximately $1 \mathrm{~mm}$ depth). Each fluorescence spectrum was corrected for wavelength-dependent excitation light intensity delivered from the excitation monochromator using an internal reference photo diode, and for the non-uniform wavelength response of the emission monochromator and PMT using factory generated corrections factors. These corrections result in the calibrated units shown in the plots below which are consistent between all data sets.

For these experiments, physical gels and chemical gels were tested. Physical gels were of the same concentration of gelatin, but had no mTG included as a control for the activity of the enzyme, and thus gelled after cooling. These gels were then brought back up to $37^{\circ} \mathrm{C}$ and tested, which corresponds to the melted gel data set. Chemical gels were created as described, with the incorporation of $\mathrm{mTG}$.

\section{Results}

\section{Verification of crosslinking and material properties}

Tensile and rheological testing of gelatin and collagen crosslinked with mTG has been analyzed previously ${ }^{13,16,22-25}$ along with gelation properties using thermal cycling. ${ }^{16,22}$ For our purposes, only the elimination of the melting point at $37{ }^{\circ} \mathrm{C}$ in physical gels (gelatin that has been gelled by cooling, without enzyme present) is necessary for the integrity of the microchannels during incubation at $37{ }^{\circ} \mathrm{C}$ for cell culture and analysis. The melting temperature of the physical gel used was verified to be at $37^{\circ} \mathrm{C}$, but the chemical gel (gelation occurs in the presence of enzyme) remained unmelted at a gel temperature $95^{\circ} \mathrm{C}$, after which point the water began to boil out of the dish surrounding the gel and the experiment was stopped. This is consistent with previous calorimetric data on collagen gels showing no melting behavior until temperatures at which the proteins begin to denature (approx. $177^{\circ} \mathrm{C}$ ).$^{16}$

Previous tensile data has shown that chemical gels similar to those we molded have significantly higher Young's Moduli than the physical gel. ${ }^{13}$ The strength and stiffness of our chemical gels have proved to be sufficient for the processes involved in molding and cell culture. Molding was done as described to produce microfluidic channels in a crosslinked gelatin matrix as shown in Fig. 2. Percentages of gelatin from $10-15 \%$ were sufficient to produce gels that were strong enough to be removed from the molds.

\section{Thermal protection and tertiary structure}

Although the amino acid sequence of gelatin is the same as that of collagen, protein chains tend to be shorter and somewhat denatured due to the processes used to produce gelatin. Tertiary structure and covalent crosslinking of proteins can often influence cellular interactions. Maintaining the tertiary structure common between collagen and gelatin is important for cell-gel interactions via cell surface receptors which can bind collagen, so that cellular response would be similar to that on pure collagen (e.g., for mammary epithelial cells to form ducts in 3-D culture, collagen IV must be present and capable of interacting with receptors ${ }^{8}$ ). Collagens form triple helices spontaneously and it is this structure that is partially responsible for the stiffness of physical gelatin gels, formed by cooling of a gelatin solution.

This triple helical structure was shown to exist in enzyme crosslinked gelatin gels, and also that it is degraded during incubation at $+70{ }^{\circ} \mathrm{C}$ for extended periods of time resulting in reduced gel strength. ${ }^{22}$ Both during commercial purification of 
gelatin and during processing for molding, thermal denaturation of the gelatin can occur. In addition, the formation of covalent crosslinked gelatin by $\mathrm{mTG}$ partially prevents the collagen-like tertiary structure from forming in the cooled crosslinked gelatin gels. ${ }^{26}$ For these reasons during molding, the gelatin solution was never heated to above $70{ }^{\circ} \mathrm{C}$, to maintain gel strength and tertiary structure during cell culture.

\section{Fluorescence behavior characterization}

The crosslinking and structure of collagen has been linked to its ability to fluoresce at $400 \mathrm{~nm}$ when excited at $320-325 \mathrm{~nm}$, due to the absence of aromatic residues. ${ }^{27}$ The presence of this type of autofluorescence in a substrate material could interfere with cellular analysis using fluorescent dyes. Because this material is to be used for cell culture and analysis, it is important to know if there are any limitations on the excitation spectrum of dyes used due to the autofluorescence of the material.

We tested the fluorescence capabilities of the gelatin in enzymatically crosslinked chemical gels, in addition to melted and unmelted physical gel samples (as controls for the effects of the enzyme on gelatin structure). Emission spectra of all three types of gels are shown in Fig. 3(a) for an excitation of $320 \mathrm{~nm}$ to determine if the gelatin had similar excitation properties as collagen. The units of fluorescence intensity are in calibrated units, and the spectrum for a cuvette full of water alone is included as a reference for the magnitude of these intensities. These data show that aspects of collagen are retained in the crosslinked gels due to very similar excitationemission spectra (excitation peak at $320 \mathrm{~nm}$, with an emission peak at $400 \mathrm{~nm}$ ).

The data in Fig. 3(a) and (b) show that the levels of autoflorescence in this material would not be expected to limit the use of dyes exciting between $310 \mathrm{~nm}$ and $330 \mathrm{~nm}$ despite the presence of some fluorescence. The fluorescence peak height is approximately three times that of the intensity due to interactions of the light with water and would be expected to fall within the normal range of background fluorescence during an experiment. Also, this peak height actually decreases with gelation and also with covalent crosslinking, as expected from the small disruption of gelatin structure after chemical crosslinking.

The visualization of cells in collagen gels is typically done using various fluorescent dyes and ideally similar techniques could be used to visualize cells in these gelatin microfluidic devices. To verify this, the autofluorescence at the excitation wavelengths of common fluorophores were tested. Fig. 3(c) shows that the emission intensities with excitations of $490 \mathrm{~nm}$ (FITC, ethidium bromide), $550 \mathrm{~nm}$ (propidium iodide, Alexa 555), and $650 \mathrm{~nm}$ (Alexa 647), are two orders of magnitude lower than the peak from $320 \mathrm{~nm}$ excitation. We conclude that no significant limitations exist for use of this material in conjunction with fluorescent dyes due to autofluorescence at common wavelengths of interest.

\section{Cell attachment and morphology assays}

This material shows promise in allowing a variety of characteristics of the cellular microenvironment (soluble and matrix) to be controlled simultaneously. As a test of cytocompatibility and scale effects, we have compared attachment and morphology of NMuMG cells in macro- and microscale cultures on both tissue culture polystyrene and on gelatin gels.

Cells were seeded on traditional tissue culture polystyrene in both macro- and microscale cultures to compare differences in behavior at different volume densities (scales) of cells grown on the same substrate type. Microscale cultures on gelatin coated polystyrene were done as a control for attachment of cells onto gelatin coated, but rigid surfaces. These conditions can then be compared to the cells grown in microscale cultures in crosslinked gelatin channels. For the microscale cultures on polystyrene (coated or uncoated), PDMS microchannels were placed on tissue culture treated polystyrene surfaces and cells seeded directly onto the polysytrene. The cells were seeded directly onto the gelatin surfaces in the molded gelatin devices. For all cultures, regardless of scale, the surface seeding density of the NMuMGs was 40,000 cells $\mathrm{cm}^{-2}$. The volume density was 20,000 cells $\mathrm{mL}^{-1}$ in the macroscale culture and 1 million cells $\mathrm{mL}^{-1}$ in the microscale cultures.

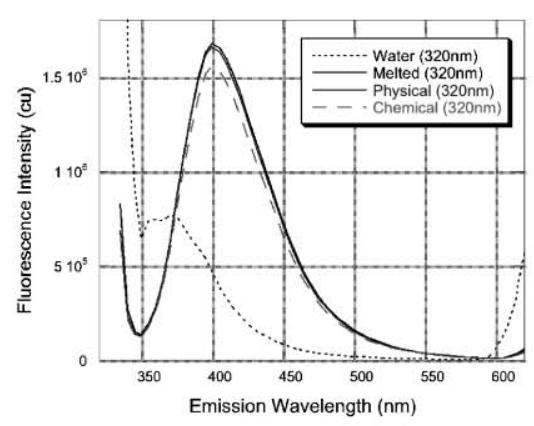

(a)

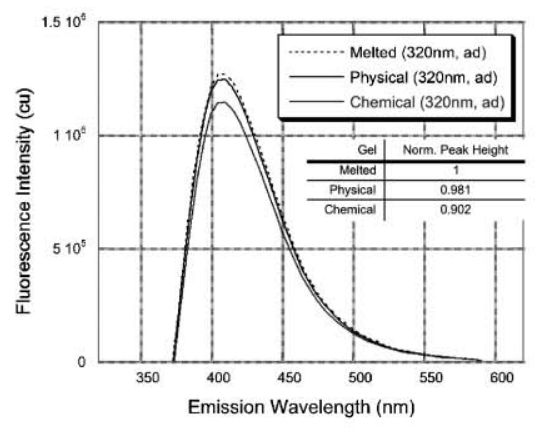

(b)

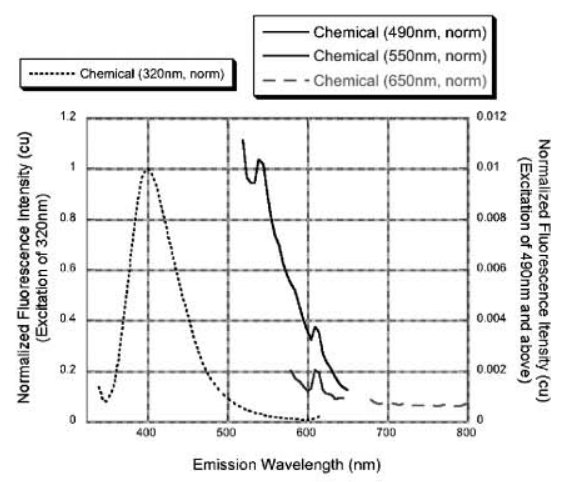

(c)

Fig. 3 Fluorescence data for different gel types when excited at $320 \mathrm{~nm}$ (a), corrected data showing just the fluorescence from the gels, with the contribution from water removed (b), and the spectra for chemical gels excited at different relevant excitation wavelengths (c). The magnitude of autoflorescence at an excitation of $320 \mathrm{~nm}$ is only three times that of water, and reduces with chemical crosslinking. The magnitudes of autofluorescence at excitations of 490,550, and $650 \mathrm{~nm}$ are two orders of magnitude smaller than that at 320 nm and would not be expected to be detectable in an assay in which fluorescent dyes are used for cellular analysis. 
The attachment kinetics and morphology of cells from the same passage were analyzed at 5 and 24 hours after initial seeding for each of the four conditions (shown in Fig. 4 for both time points). The results for both the macro- and microscale cultures on polystyrene or gelatin coated polystyrene showed monolayaer cell layers that had begun to attach to the rigid surfaces at 5 hours (Fig. 4, top panel, (a), (b) and (c)). Cells remained circular in the gelatin gel channels unlike the monolayer cultures seen in the three control conditions, but did not appear to be undergoing apoptosis (cells looked bright and solid). This cell line is adhesion dependent, thus if these cells were in fact not in the process of attaching to the substrate properly, high rates of apoptosis would be seen in less than 24 hours resulting in a significant decrease in cell density and the elimination of any intact cells in the channels.

The cell status at 24 hours was then analyzed visually to ensure that these cells were attaching to the substrate and surviving (Fig. 4, lower panel). Densities of both macro- and microscale cultures on polystyrene were similar and the cells remained in typical monolayer cultures (regardless of surface treatment). Cells in the gelatin gel microchannels however,

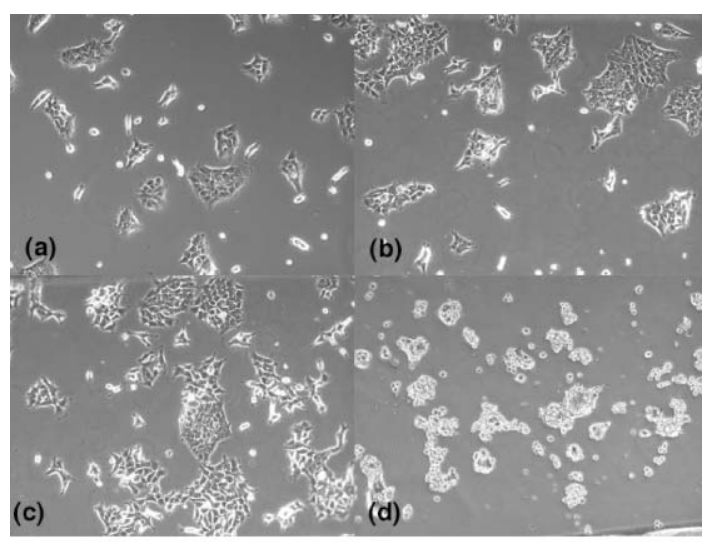

5 hours

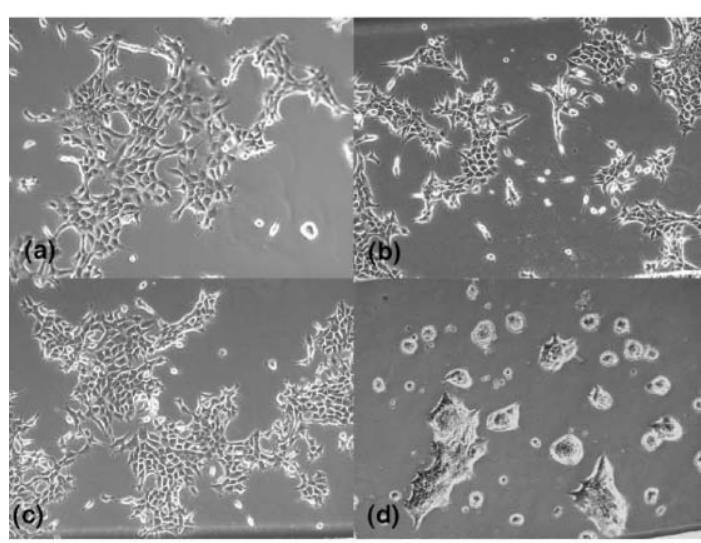

24 hours

Fig. 4 Representative phase contrast images $(20 \times)$ of NMuMG cells grown in various culture conditions at 5 hours and 24 hours after seeding (using an inverted microscope, visualizing cells through the substrate). In both panels, macroscale cultures on tissue culture plastic are shown in (a), microscale cultures on tissue culture plastic in (b), microscale cultures on a gelatin coated surface in (c), and microscale cultures in crosslinked gelatin microchannels in (d). showed the development of 3-D structures, instead of spreading and forming a monolayer as in the other conditions. The focal plane of the microscope could be set to the channel bottom, and by focusing downward into the gels, the images of the cells came into focus, indicating that they had begun to invade the matrix. This resulted in the halos seen in Fig. 4, (lower panel, (d)), in phase contrast that are not seen in the monolayer cultures. If these cells were in fact not attached to the substrate, by this timepoint all the cells would have undergone apoptosis and no cells would be seen in phase contrast. Additional verification of the 3-D structure was performed by staining nuclei and the cytoskeletons of these structures and comparing these images to those obtained from cultures on 2-D rigid substrates (shown in the ESI, Fig. 5†). Also, long term cultures were maintained to verify that cells could proliferate and survive on the gelatin gels for longer than 24 hours. Phase images after 3 days were taken for gelatin gel cultures and for rigid controls and are shown in the ESI, Fig. 6.†

In these gelatin microdevices, these cells can form 3-D structures that invade down into the surfaces of the gelatin microchannels, allowing fluidic contact from above and 3-D culture simultaneously. Results from other groups using this cell line in 3-D collagen gels (unmolded) have shown that mammary ducts can be produced, with initial results that are very similar to those seen in Fig. 4 for the gelatin gel microchannels at 24 hours. $^{8}$ Wozniak et al., showed that in collagen gels, cell proliferation is balanced with tubulogenesis (for mammary cells) and matrix stiffness can affect this balance via cell signalling cascades (through Rho and ROCK mediated contractility). Thus, assays of proliferation (or lack thereof) in a system such as this are not necessarily comparable to the 2-D cultures and so we must rely on morphological assays. Future work will focus on the analysis of the signalling cascades active in these cells in different microenvironments.

\section{Discussion}

Several synthetic matrices that have recently been explored all aim to simulate the extracellular matrix, as its importance in many different systems is beginning to become clear. ${ }^{28-30}$ The mechanical aspect of the matrix is important for the development of correct tissue structure (e.g. formation of a basement membrane as a substrate for polarization of epithelial cells ${ }^{31}$ ) and mechanotransduction (e.g. cell stiffening or increased contractility in response to externally applied forces $^{32}$ ). Thus, a rigid polymer substrate, even when coated with ECM components, does not mimic the mechanical aspects of the in vivo environment.

Chemically, the ECM is important for providing access of cells to growth and differentiation factors, and also for interacting with cell surface receptors such as integrins, affecting many signaling pathways within the cell. These interactions can be disrupted in vitro when ECM components adsorb onto surfaces at unknown concentrations and in unknown conformations (and are potentially denatured). ${ }^{33}$ Also, the matrix is not simply a passive reservoir for soluble factors to be used when the cell chooses, but actively participates in the transport and regulation of these 
substances. ${ }^{34}$ Important growth factors are not only regulated at the level of production, but also at the level of actual availability to cells both spatially and temporally via ECM mediated regulation. This role is completely absent in traditional cultures.

When the mechanical and chemical roles of the ECM are combined with the soluble portion of a cell's in vivo microenvironment, an immensely complex system of regulation and signaling emerges. Tissue culture media are generally mixtures of defined (such as growth factors like insulin) and undefined (such as fetal bovine serum) components that have been found to improve results.

Similarly, products are available to aid in cell attachment that are used by coating the substrate with ECM proteins obtained from tissue (e.g., bovine, murine), however the components of these treatments are not well characterized. For example, Matrigel is a common surface coating material isolated from the EHS mouse tumor and is described by the manufacturer as having various ECM proteins, a variety of matrix metalloproteinases, growth factors, and other "undefined components" established by limited studies done over a decade ago. ${ }^{35}$ Although these components are found in vivo (in a murine tumor), their concentrations and activities in the cellular microenvironment in in vitro macro- or microscale cultures are not known.

The ability to not only control the soluble portion of a cell's microenvironment via microfluidic channels, but also the composition of the matrix is a step towards being able to define the necessary components for cellular behavior. The devices created could be used with defined media and have only specific components in the matrix on which the cells are seeded, thus removing many layers of complication from traditional in vitro cultures.

The response of the NMuMG cells cultured in the microfluidic channels fabricated using this protocol was very different on the flexible, degradable protein surface than those on the rigid surfaces regardless of the presence of ECM proteins as a coating. In vivo, these cells form branching duct structures that fill the flexible and deformable mammary fad pad, not a flat sheet on a rigid surface as in traditional cultures. We propose that the development of more 3-D structures in the gelatin based microfluidic channels is a more in vivo-like response than that of the cells on the other rigid substrate controls where the cells formed a monolayer. These results suggest that microenvironments created in gelatin microfluidic channels may be more similar to the in vivo conditions than traditional culture and could potentially allow for new types of analyses of cellular behavior and function.

An important aspect of these devices is that they contain no synthetic compounds used during fabrication, ensuring biocompatibility and the purity of the microenvironments used for cell culture. Synthetic polymers such as poly(lactic-co glycolic acid), (PLGA) have been polymerized into biodegradable cell scaffolds for bone regeneration among other applications, and bioactive materials can be grafted onto the polymer to improve its cellular interactions. ${ }^{36}$ Other engineered cell scaffolds or adhesives using synthetic polymers have used photoinitiators in order to make photodefinable polymers, or to polymerize the material in situ. ${ }^{29,30}$
However, the presence of toxic compounds used for fabrication limits the use of these scaffolds in microfluidic devices where the effects of the microenvironment are of interest. Thus, organic solvents, porogenic compounds and photoinitiators, although normally used at low concentrations in these systems, can be very cytotoxic in the small volumes of a microfluidic device. ${ }^{10}$ Other techniques such as electrospinning of polymers ${ }^{28}$ or proteins ${ }^{37}$ create functional cell scaffolds, although cells must be added after fabrication and allowed to migrate into the matrix (potentially long distances, on the order of millimeters) instead of being incorporated during fabrication throughout the material. When short-term effects of microenvironment on cellular behavior are of interest or when cells are used that do not survive for long periods in vitro such as many primary cells, these types of fabrication systems would not be suitable.

\section{Conclusions}

We have fabricated all natural protein-based microdevices using a biological enzymatic crosslinking process. Tranglutaminase crosslinked gelatin microdevices allow photodefinable geometries to be molded into a completely natural biopolymer with no synthetic components. These microfluidic devices were shown to be cytocompatible for microscale cultures of murine mammary epithelial cells (NMuMG). Cell culture results show that gels can allow three dimensional cell growth and expansion while still maintaining fluidic access to the cells. Also, the autofluorescence of the material was shown to be of very low intensity with the highest peak at $320 \mathrm{~nm}$ excitation and exhibited only levels three times that due to interactions of the light with water.

Cell culture results suggest that this platform may not only provide a more in vivo-like in vitro culture environment (in visio), but also allow for a wider range of readouts that potentially are more physiologically relevant. Rather than expression profiles and proliferation assays, actual functionality such as matrix invasion and duct formation could be measured. In addition, due to the low autofluorescnce of the material, the use of fluorescence to visualize the 3-D structures formed by these cells will allow further analysis of structures and distributions of different cell types.

Morphological readouts may also allow for new types of analyses of invasive (metastatic) or migratory cell types. Currently, most migration and invasion assays are done in 2-D tissue culture type setups, (some include a layer of ECM components on the rigid polymer membrane), which are subject to the same limitations as any traditional in vitro assay. This particular material and fabrication process, in conjunction with microfluidics could provide new platforms for assays involving cell migration and attachment that could be performed in more controlled, in visio environments, allowing more physiological readouts to be analyzed.

\section{Acknowledgements}

The authors would like to thank Dr Greg Payne and Dr LiQun Wu for very useful discussions and also providing enzyme for the project. Also, Dr Patricia Keely for assistance with 
imaging and insight into working with three dimensional cell structures.

\section{References}

1 D. Aeschlimann and V. Thomazy, Protein crosslinking in assembly and remodelling of extracellular matrices: the role of transglutaminases, Connect Tissue Res., 2000, 41, 1, 1-27.

2 S. Raty, E. M. Walters, J. Davis, H. Zeringue, D. J. Beebe, S. L. Rodriguez-Zas and M. B. Wheeler, Embryonic development in the mouse is enhanced via microchannel culture, Lab Chip, 2004, 4, 3, 186-190.

3 H. Yu, I. Meyvantsson, I. A. Shkel and D. J. Beebe, Diffusion dependent cell behavior in microenvironments, Lab Chip, 2005, 5, 1089-1095.

4 M. S. Condon, The role of the stromal microenvironment in prostate cancer, Semin. Cancer Biol., 2005, 15, 2, 132.

5 E. H. J. Danen and A. Sonnenberg, Integrins in regulation of tissue development and function, J. Pathol., 2003, 200, 4, 471-480.

6 W. Tan and T. A. Desai, Microfluidic patterning of cellular biopolymer matrices for biomimetic 3-D structures, Biomed. Microdev., 2003, 5, 3, 235-244.

7 M. R. Dusseiller, D. Schlaepfer, M. Koch, R. Kroschewski and M. Textor, An inverted microcontact printing method on topographically structured polystyrene chips for arrayed micro-3D culturing of single cells, Biomaterials, 2005, 26, 29, 5917-5925.

8 M. A. Wozniak, R. Desai, P. Solski, C. Der and P. J. Keely, ROCK-generated contractility regulates breast epithelial cell differentiation in response to the physical properties of a threedimensional collagen matrix, J. Cell Biol., 2003, 163, 3, 583-595.

9 G. Walker, G. Mensing and D. Beebe, Physics and applications of microfluidics in biology, Ann. Rev. Biomed. Eng., 2002, 4, 261-286.

10 G. Walker, H. Zeringue and D. Beebe, Microenvironment design considerations for cellular scale studies, Lab Chip, 2004, 4, 91-97.

11 J. Atencia and D. Beebe, Controlled microfluidic interfaces, Nature, 2005, 437, 648-655.

12 A. Ito, A. Mase, Y. Takizawa, M. Shinkai, H. Honda, K. Hata, M. Ueda and T. Kobayashi, Transglutaminase-mediated gelatin matrices incorporating cell adhesion factors as a biomaterial for tissue engineering, J. Biosci. Bioeng., 2003, 95, 2, 196-199.

13 M. K. McDermott, T. H. Chen, C. M. Williams, K. M. Markley and G. F. Payne, Mechanical properties of biomimetic tissue adhesive based on the microbial transglutaminase-catalyzed crosslinking of gelatin, Biomacromolecules, 2004, 5, 4, 1270-1279.

14 G. F. Payne, personal communication, 2005, College Park, MD.

15 L. Lim, Y. Mine and M. Tung, Barrier and tensile properties of transglutaminase cross-linked gelatin films as affected by relative humidity, temperature and glycerol content, J. Food Sci., 1999, 64, 4, 616-622.

16 A. Mizuno, M. Mitsuiki and M. Motoki, Effect of transglutaminase treatment on the glass transition of soy protein, J. Agric. Food Chem., 2000, 48, 8, 3286-3291.

17 S. G. Priglinger, C. S. Alge, A. S. Neubauer, N. Kristin, C. Hirneiss, K. Eibl, A. Kampik and U. Welge-Lussen, TGF-beta 2-induced cell surface tissue transglutaminase increases adhesion and migration of RPE cells on fibronectin through the gelatinbinding domain, Invest. Opthalmol. Visual Sci., 2004, 45, 3, 955-963.

18 A. M. Belkin, S. S. Akimov, L. S. Zaritskaya, B. I. Ratnikov, E. I. Deryugina and A. Y. Strongin, Matrix-dependent proteolysis of surface transglutaminase by membrane-type metalloproteinase regulates cancer cell adhesion and locomotion, J. Biol. Chem., 2001, 276, 21, 18415-18422.

19 T. H. Chen, D. A. Small, M. K. McDermott, W. E. Bentley and G. F. Payne, Enzymatic methods for in situ cell entrapment and cell release, Biomacromolecules, 2003, 4, 6, 1558-1563.

20 R. N. Chen, H. O. Ho and M. T. Sheu, Characterization of collagen matrices crosslinked using microbial transglutaminase, Biomaterials, 2005, 26, 20, 4229-4235.

21 G. Palmer, C. Marshek, K. Vrotsos and N. Ramanujam, Optimal methods for fluorescence and diffuse reflectance measurements of tissue biopsy samples, Lasers Surg. Med., 2002, 30, 191-200.

$22 \mathrm{H}$. Babin and E. Dickinson, Influence of transglutaminase treatment on the thermoreversible gelation of gelatin, Food Hydrocolloids, 2001, 15, 3, 271-276.

23 E. P. Broderick, D. M. O’Halloran, Y. A. Rochev, M. Griffin, R. J. Collighan and A. S. Pandit, Enzymatic stabilization of gelatin-based scaffolds, J. Biomed. Mater. Res. Part B-Applied Biomaterials, 2005, 72B, 1, 37-42.

24 V. Crescenzi, A. Francescangeli and A. Taglienti, New gelatinbased hydrogels via enzymatic networking, Biomacromolecules, 2002, 3, 6, 1384-1391.

25 T. Chen, H. Embree, E. Brown, M. Taylor and G. Payne, Enzymecatalyzed gel formation of gelatin and chitosan: potential for in situ applications, Biomaterials, 2003, 24, 2831-2841.

26 S. Giraudier, D. Hellio, M. Djabourov and V. Larreta-Garde, Influence of weak and covalent bonds on formation and hydrolysis of gelatin networks, Biomacromolecules, 2004, 5, 5, 1662-1666.

27 N. Ramanujam, Fluorescence Spectroscopy in vivo, in Encyclopedia of Analytical Chemistry, ed. R. Meyers, John Wiley \& Sons Ltd., Chichester, 2000, pp. 20-56.

28 W. J. Li, R. Tuli, C. Okafor, A. Derfoul, K. G. Danielson, D. J. Hall and R. S. Tuan, A three-dimensional nanofibrous scaffold for cartilage tissue engineering using human mesenchymal stem cells, Biomaterials, 2005, 26, 6, 599-609.

29 C. F. Tu, Q. Cai, J. Yang, Y. Q. Wan, J. Z. Bei and S. Wang, The fabrication and characterization of poly(lactic acid) scaffolds for tissue engineering by improved solid-liquid phase separation, Polym. Adv. Technol., 2003, 14, 8, 565-573.

30 X. H. Wang, W. J. Wang, D. P. Li, Q. L. Feng, F. Z. Cui, Y. X. Xu and X. H. Song, Surface modification of biodegradable PLGA scaffold with chitosan for cell culture, inFunctionally Graded Materials Vii., 2003, pp. 341-345.

31 C. H. Streuli, N. Bailey and M. J. Bissell, Control of mammary epithelial differentiation-basement-membrane induces tissuespecific gene-expression in the absence of cell cell-interaction and morphological polarity, J. Cell Biol., 1991, 115, 5, 1383-1395.

32 M. A. Wozniak, K. Modzelewska, L. Kwong and P. J. Keely, Focal adhesion regulation of cell behavior, Biochim. Biophys. Acta, 2004, 1692, 2-3, 103-119.

33 U. Hersel, C. Dahmen and H. Kessler, RGD modified polymers: biomaterials for stimulated cell adhesion and beyond, Biomaterials, 2003, 24, 4385-4415.

34 F. Rosso, A. Giordano, M. Barbarisi and A. Barbarisi, From cellECM interactions to tissue engineering, J. Cell. Physiol., 2004, 199, 2, 174-180.

35 Beckton-Dickinson, Product Catalog: Product number 354234 , Matrigel, www.bd.com, 5/17/2005.

36 H. Shin, S. Jo and A. G. Mikos, Biomimetic materials for tissue engineering, Biomaterials, 2003, 24, 24, 4353-4364.

37 K. J. Shields, M. J. Beckman, G. L. Bowlin and J. S. Wayne, Mechanical properties and cellular proliferation of electrospun collagen type II, Tissue Eng., 2004, 10, 9-10, 1510-1517. 